\title{
ÜBER DIE MARGARETENLEGENDEN.
}

Die Margaretenlegende verbreitete sich schon fruh vom orient, ihrem schauplatze und entstehungsorte, auf dem wege durch Griechenland über das ganze abendland. In Deutschland tritt sie zuerst bei Hrabanus auf, der in seinem martyrologium*) schon die hauptztige derselben berichtet. Einer wie allgemeinen beliebtheit sie sich erfreute, zeigen besonders die mannigfachen poetischen bearbeitungen, die sie seit dem 12 . jahrhundert in Deutschland, England, Frankreich in den nationalsprachen erfullr. Nicht als ob man den inhalt der legende als besonders dazu geeignet hätte ansehn müssen - so anziehend auch wenigstens dem verdorbenen geschmacke der späteren zeit das auftreten des teufels in derselben gewesen sein mag - vielmehr der besonderen geltung der heiligen ist das zuzuschreiben. Margarete war die christliche Lucina, wie sie Hieronymus Vida**) auch geradezu anredet: das gebet zu ihr, das lesen und verbreiten ihrer passion verhalf den frauen zu gltucklicher niederkunft; das wird ausdrücklich in der legende erwähnt und segen fur kreissende folgen unmittelbar auf dieselbe in mehreren handschriften.

Deutsche poetische Marraretenlegenden sind schon in mannigfaltiger gestalt und nicht geringer anzahl bekannt gemacht. Die älteste unter ihnen ist wol die in Haupts zeitschr. I, $152 \mathrm{ff}$. veröffentlichte: mehrere nur noch im 12. jh. mögliche reime, welche das gedicht aufzuweisen hat $\left.{ }^{* * *}\right)$, beweisen, dass dieje-

*) Opera VI. 190. F. G.

**) opera Antverpiae 1578. s. 176. v. 49.

**) edele: Eugìne 113, ubcle: himele 247, himele: nidene 633, Margarête: guote 14; das letztere in der hs. nur hier, aber sonst oft mit Haupt herzustellen, am sichersten v. 149, 443, 449, 461, 827, 6\$9, 743, 715. 
nigen, welche das gepräge einer jüngeren zeit tragen*), dem originale nicht angehört haben können. Uebrigens scheint die hand des uberarbeiters auch sonst erkennbar: so v. 83 u. 84, wo der dichter aus der schaar der heiligen jungfrauen allein die "frouwe Elspet" namentlich hervorhebt. Damit kann die mutter Johannes des täufers nicht gemeint sein, die sehr wenig als heilige verehrt wurde, wenigstens enthalten weder die vitae sanctorum des Surius noch die legenda aurea in ihrer ursprïnglichen gestalt die geschichte derselben (die Acta SS. sind noch nicht bis zu ihrem heiligentage gekommen). Eine solche verehrung, um vor allen andern heiligen genannt zu werden, genoss nur die heil. Elisabeth v. Thüringen (1235 canonisiert); die verse sind also späterer zusatz. - Noch in dasselbe jahrhundert setzt Bartsch die nach einer Prager hs. des 15. jh. Germania 4, 440 von ihm herausgegebene "Nargareten marter" (Varianten einer Klosterneuburger hs. Germ. 6, 376 von J. M. Wagner). Auch hier finden sich reime, die doch jener zeit schwerlich zuzutrauen sind. Beispiele für apokope des $e$ nach langer stammsilbe und für unorganische dehnung des kurzen stammvokals eines zweisilbigen wortes kommen freilich schon in reimen der gedichte des 12 .jhs. vor, aber doch nur ganz vereinzelt. Weinhold bair. gramm. $§ 338$ führt als beispiel für apokope erbetail dat. (:siptail n.) und bluot dat. (:tuot) denkm. XLVI, 40 u. 80 und einige belege für denselben fall aus Wernhers Maria und Dietmar v. Aist an; weniges lässt sich aus älteren gedichten hinzufügen: eine härtere apokope erheischt der reim sache: ungemach**) Diem. d. ged. 48, 17 (buicher Mose), gleichfalls die des $e$ des dat. m. der reim sinne: chint a. a. 0. 87, 22 (in dem gedichte diu wârheit). Verletzung der quantität findet sich ebenfalls schon in den bb. Mos. 24, 24 im reime ôle: wole und in der jüngern Judith Diem. 160, 14 im reime frâgen:sagen. Aber was haben solche seltenheiten $\mathrm{zu}$ bedeuten gegentiber einem gedichte, in dem jeder zehnte vers eine solche apokope des $e$ nach langer stammsilbe erfordert und noch dazu darunter die des $e$ des plur. (v. 342) und des dat. fem. (307)? Das

*) so 109 land:sant, 167 christ:frist (conj.), 324 sin:minne, 526 hass (dat.): vass, 561 wild (willst): pild; 703 slahen: gâhen, 555. 697 u. a.

$\left.{ }^{* *}\right)$ Wenn man nicht lieber in ungemach anfügung eines unechten $c$ annehmen will vgl. Weinhold b. gr, § 342 . 
darf man einem österreichischen dichter des 14 . jhs. ohne bedenken, möglicherweise auch einem des 13., keinesfalls aber cinen friberen zutrauen. Wenn sich nun aber doch daneben altertimliche reime wie heiligon (đ. pl.): lôn (v. 3), guote: Margarête (v. 11) finclen - es kommen noch einige weniger sichere hinzu - und wenn der ganze frische, knappe und einfache stil für das 12 . jh. zu sprechen scheint, so werden wir auch hier eine grundlaye aus dieser zeit reraussetzen missen, wie das gedicht uns aber vorliegt, ist es eine spätere uberarbeitung. Die annahme einer ältern grundlage könnte auch dadurch noch gestiitzt werden, dass gerarle an mehreren bedenklichen stellen die beiden hss. nicht uibereinstimmen so v. 259 u. 60,307 u. 8, 419 u. 20; in antern fällen zeigen sich abweichungen von der lateinischen quelle, so v. 339, wo der reim chragen:lâgen zwar auch in der Prager lis. beibehalten werden muss wegen der lesart der Klosterneuburger oben fif sinem chragen zwei prinnunde swert ligen; aber die quelle hat: super collum ejus erat serpens, gladius candens in manu ejus videbatur; es scheint also hier schon die vorlage beirler hss. entstellt gewesen zu sein.

Auch im 13. jh. wurde dic Margaretenlegende poetisch bearbeitet. Ein freund des Rudolf v. Ems, Wetzel, hat nach des ersteren bekannter angabe im Alexander M. S. 4, 867 "vil gefuoge" ein Margaretenleben verfasst. Die identität desselben mit einer nur teilweise in einer hs. des 15. jhs. erhaltenen Margaretenlegende, die der herzogin Clemende von Zähringen gewidmet ist, hat Bartsch, germanistische studien I, $1 \mathrm{ff}$, nachzuweisen gresucht. Wem sich auch dariber eine absolute gewisheit aus den wegebenen anhaltspunkten nicht gewinnen lässt, so macht es die ron Bartsch grerchene zeitbestimmung doch immerhin wahrseheinlich und jedenfalls ist dies durchaus im höfischen stile des 13. jhs. gehaltene gedicht das einzige unter den zahlreichen bekamiten Marqaretenlegenden, welches auf Wetzcls autorschaft anspruch machen kann *). Höchstens könnte vielleicht noch die von Hoffimam Wiener hss. n. 35, 45 angefiihrte bearbeitung in betracht kommen, die möglicherweise noch im 13. jh. von einem Oherdeutschen verfasst wurde, doch

*) Ich fiihre laher in folgenclen auch das gedicht unter Wetzels namen an. 
gestatten natürlich die beiden dort mitgeteilten anfangsverse*) nicht einmal darliber einen schluss. Die hs. ist aus dem 14. jh. - Sonst gehört ins 13. jh. nur noch die im Passional enthaltene Margaretenlegende (bei Köpke s. $326 \mathrm{ff}$.) und wol auch das mitteldeutsche gedicht von einer menlichen iuncvrowen, dessen eingang von Lappenberg im anzeiger f. kunde der d. vorz. III, 39, aus einer Hamburger hs. des ${ }_{n} 13$. oder 14. “ jhs. mitgeteilt ist**).

Dem 14. jh. gehört die Margareta an, welche Docen dem Hartwig rom Hage, dem dichter der 7 tagzeiten (Hagens museum II, 265; hs. v. j. 1348), zugewiesen hat und woron er altd. wälder III, 149 ff. proben mitteilte.

Hierher, schwerlich in frühere zeit, ist auch Margarêten passîe in Schades geistl. gedd. des 14. und 15. jhs. vom Niederrhein zu setzen. Schade vermutete zwar, dass diesem gedichte ein früheres des 12. jhs. zu grunde gelegen habe. Das hat sich auch insofern bestätigt, als der niederrheinische dichter die oben besprochene Margareten marter des 12. jhs. benutzt hat. Aber er entnahm ihr wenig mehr als den anfang der eigentlichen erzählung (cf. Bartsch Germ. 7, 268 f.) und arbeitete im übrigen selbständig nach einer vielfach abweichenden lateinischen quelle. Das geschah aber schwerlich eher, als im 14. jh. denn während von den unreinen reimen keiner notwendig auf das 12. jh. zurückzuführen ist, können die reime mer (mare): wer (esset) v. 223, gedeuft (part.): geleuft (praet.) v. 304, dede (conj.): gebede 336 nur von einem späteren dichter herruhren.

Noch nicht bekannt war bisher meines wissens eine niederdeutsche bearbeitung der legende, welche in einer der Göttinger univ. bibl. angehörigen papierhs. des 15. jahrhunderts (Cod. M. S. theol. 199. $8^{0}$ ) enthalten ist. Die hs. (25 bll. in kl. 8) enthält auf bl. 1-23 a die Margaretenlegende ohne tiberschrift; daran schliessen sich unmittelbar bl. $23^{a}-24^{a}$, Vota ad par-

*) Die edel magt hoch geporn mit allen tvgenden auzerchorn

**) Ez was ein iuncrrowe guit An gůten worten wol behuit Di hette riche mage $\mathrm{Si}$ was in schoner plage Wol gewaszen als de kint De schone vnde lep sint. 
tum mulieris, ut pariat absque gravi dolore*) «, worauf bis zum schluss eine niederdeutsche ibertragung der ersten $14 \mathrm{vv}$. des ev. Joh. folgt.

Den von hochlleutscher einmischung nicht ganz freien dialekt wird folgende übersicht des lautbestandes kennzeichnen.

\section{Vocale:}

hd. $a=e$ in nesze (nase), der (audeo). vor $l=o$ in oll, koldes.

" $\hat{a}=o$ vor ch : brochte, noch.

" schwàches $e=i$ : martir, andirs, godis: $=0$ in vor-.

$\eta \ddot{e}=u$ in sulven, hulpe.

$\rightarrow \hat{e}=i$ in irsten (primum), sile;

$=0$ in or (ehe).

n $i=u$ in sulverin, ummer;

$=e:$ hen, hemmel, em, er, met, beth, weten, wedder;

$=o:$ or (ihr, neben er).

, $o=a:$ van, ader, salt, laüe, gade (selten).

, $u=0:$ koningk, joden, dorch, or-, borde, worde.

,$\quad x=e$ : gebere, slede (siehe unten uiber den umlaut).

" ei=ei (ey): eyn, bereydet, geheiten;

$=e:$ menden, schen, bleck, weck, wesze (waise);

$=i$ : hiliger, hilgen.

$\eta \quad i e=i:$ lith, hil, gingk, hingk, rypen, kyszen;

$=e:$ (selten) derst.

n $i u=u: l u d e$, du/el .

"ou= o : dope, lope, houet, rock; = ou vor $w$ : frouwen, schouwen und in ougen.

n $u o=0: k o p e$, moder, mol, tho; bokelin, vote;

$=u:$ vluck, ge/uch, gule, hu/f, suchten.

Der umlaut von $a$ ist mit wenigen ausnahmen (z. b. irfaresulu iherall durehgedrungen, selbst der von $a$ wird meist durch $e$ bezeichnet, daneben aber z. b. salich. Alle andern vokale kennen den unlaut nicht und ich habe deshalb der einfachheit halber im vorstehenden die ungelauteten mhd. vocale hier unter dic unumgelauteten mit einbegriffen.

\section{Consonanten:}

hd. $b$ inlautend $=v(u)$ : vordryven, louen, suluen.

*) Wie aus diesem an sich wertlosen lateinischen gebete hervorgeht, wurde die hs. fiir eine trau namens Anna verfasst. 
hd. $p$ auslautend $=f$ : hu/f, wiff, liff.

$n$ pf anlautend $=p$ : plegen; inlautend $=p p$ : appel.

$n$ verschobenes $f$ in- und auslautend $=p$ : hulpe, hope; warp, up.

n $t=d:$ dufel, dede, godis; daneben, wenn auch sclten, $=t$ : gute, gote, trost, teil; apokopiert in is.

${ }_{n} z=1$, ausser in czart, czeler, zcirhcit, crucze, kercze und einmal: hercze (: kercze).

n $g=c h$ vor $t$ in licht (liget)

"c im auslaut $=$ ch: mach, ge/uch.

"verschobenes $c h=k$ : bolelin, siken, vhuck, welk; daneben selten = ch: suchten (neb. sokeden), sprach.

$h$ fällt hie und da zwischen vocalen aus: sie (video), vlie.

Verdoppelung des consonanten nach kurzem vocal findet sich in mennigher, hemmel, numme, ummer, wedder, leddich u. a.

In betreff der flexion ist zu bemerken: für die verbalendungen das $n$ des pl. praes. ind. (gy geven); für den ablaut das $e$ des plur. und der 2. sing. in ind. praet. der 2. ablautsreihe (gevest, breken). Die formen der personal-pronomina sind die gewöhnlichen niederdeutschen. Der artikel schwankt im nom. sing. (u. plur.) m. u. f. zwischen die $d y d e^{*}$ ); entsprechend lautet das interrogativum $w y$, wie. Dem mhd. nehein entspricht neyn.

Zu den eigentümlichkeiten der niederdeutschen schreibweise gehört die anwendung des $z$ und $s z$ für $s$ und die bezcichnung eines gedehnten vocals durch nachgesetztes $e$, die jedoch nur selten angewant wird (fuer feuer, gruet gruss, bluet), einmal wird auch $i$ dafur geschrieben (vorgoil vergoss). Oefter kommt das $e$ ther dem vokal vor, in welchem einige das zeichen fur den umlaut geschn haben. In unsrer hs. sind die beispiele (lafür folgende: $\left.{ }^{* *}\right)$ nót, gebith (pract.), dóth (tut), dót (tod), vít, mót (muss), gót (goss), vóp (wuof), bóck, róst, or (ehe), or (ihr, dat. f.), gót (gott); behút, mút (muss), múth (animus); wit, nèt (hasste), lith (liess), rip (ricf), vil (cecidit), $n y$ (nie); scháp; maýt. Dass also wenigstens hier dies zeichen keinen umlaut anzeigen kann, liegt auf der hand: man müste denn annehmen, dass

*) einmal auch der n. sg. m.

**) Hier, wie im folgenden immer, wird vocal mit übergeschriebenem e durch vokal mit strich widergegeben. 
das ud. für o und $u$ nur falschen umlaut gekannt habe. Deshalb braucht man aber dies $e$ keineswegs für bedeutungslos anzuschn: der schreiber scheint mir einfach dasselbe damit zu bezeichnen wie mit dem seltener nachgesetzten $e$, nämlich die länge des betr. rocals. In allen beispielen sind wir berechtigt langen vocal anzunehmen ausser in dem einzigen gót (gott), was sonst immer got geschrieben wird, und dass hier der schreiber sich versehn habe, der noch weit schlimmere dinge machte (z.b. morder für moder schrieb), ist wol keine zu kühne annahme. Bemerkenswert ist noch, dass das zeichen nur da angewant wurde, wo die quantität zweifelhaft sein konnte, also nur vor auslautendem, nicht vor inlautendem einfachen consonanten, denn im letzteren falle war die lïnge des rorausgehenden vocals selbstverständlich *); also: mút, mót, aber conj. mute, mote; bóck aber bokelin. In mayjt und ny - auch mayet und nye - geschrieben - wurde wol das e gesprochen.

Ich lasse den anfang des gedichtes folgen. Die abbreviaturen habe ich aufgelöst und interpunction hinzugefügt, sonst nichts geändert.

ET scholen alle frouwen 1 a

Die martir gerne schouwen,

Horen vnde lezen

Vnde stede dar nha weszen,

5 Alze margarete dy reyne mayt,

Dar von vns dit bokelin sagit.

Sic kan wol vordryven dy nót,

Die gót suluen gebóth,

Dar van id eiien miszgegingk:

10 Don sic den appel vnme vingk,

'Tho hant wart dy vluck gedan,

Dy noch den frouwen henget an.

Die vluck tweyerley wart:

(ingt les irsten nye vorgath:

15 He sprack du salt weszenn

Under dynes mannes bezem.

Szo isz dat dy ander vluck

(Nutte isz ock syn gefuch),

Dat sie des mannes borde

*) Die gemination des folgenden consonanten, welche hier bei vorausgehendem kurzen vokal eintrat, wird freilich ebensowenig consequent vom schreiber bezeichnet wie im erstern falle die lïnge des vocals vor auslautendem consonanten. 
5 Nummer leddich worde

Ane pyne vnde ane noth.

Dar van lidet mannich den doth.

Isz dat sie geneszenn

Vnde tho hulpe kyszen

10 Margareten die reyne mayet,

Dar van vns dit bokelin saget.

Or vader was eyn heyden,

Van gode was he gescheiden.

Don sie van em geborn wart,

15 He szande sie dorch syn hath

Auer vefteyn milen in eyne stath,

Dar ynne he eyne amme bath,

Dat sie des kindes scholde plegen

Vnd were em wol gewegen.

Mit diesem eingang stimmt vom dialekt abgesehn, fast wörtlich der anfang einer Margaretenlegende überein, welchen Hagen im grundriss s. 279 mitteilt bis auf die vv. s. 2, 1-8 bei Hagen. Zwischen beiden steht hier ein Leipziger druck vom j. $1517^{*}$ ), welcher in den vv. Hagen s. 2, 1-8 und teilweise auch in dem von der nd. bearbeitung abweichenden schlusse mit Hagens Margarete übereinstimmt; da dieser druck im tibrigen teile des gedichtes mit wenigen abweichungen den text der Göttinger hs. - nur hochdeutsch - repräsentiert, so können wir den schluss ziehen, dass diese 3 bearbeitungen zuletzt auf eine grundlage zurtickgehen. Dazu kommt dann noch die von Docen Altd. wälder III, 156 erwähnte legende, welche im wesentlichen mit der Hagenschen ubereinstimmt, ferner, wenn man nach den beiden ersten vv. schliessen darf, auch die in einer Olmutzer hs. des 15. jhs. enthaltene, welche Bartsch, anzeiger für kunde der d. vorz. 1861. s. 331 , anführt und die Margareta einer Wiener hs. (Hoffmann Wiener hss. n. 90, 2), deren anfangsworte mit denen der letzterwähnten völlig ilhereinstimmen.

Endlich aber erweist sich nun auch noch das von W. Schum in der Germania 18, 98 ff. veröffentlichte Erfurter bruchstuck einer gereimten Margaretenlegende als hierher gehörig.

*) 'Sant Margaretha legend.' Auf dem titel ist die heilige mit einem buche und einem kreuze in der hand abgebildet den drachen am bande haltend. 
Man vergleiche:

G. (Güttinger hs.) 19a, 5. Beelczebuck bin ick genant Mynen gesellen bin ick wol bekant Dy lude ick ouer wynne Er gebet ick gar vorslinge Ick mit allen rechte Vnde alle myn geslechte Nymant my ouer wynnen mach Du bist my eyn grot wedder slach 0 we myn geselle Bosze is vnsze geuelle.
E. (Schums bruchst.)

1 Belczebugk byn ich genant Mynen gesellin ben ich wol bekant Dy lute ich ubirwinde Or gebete ich gar vorslynge

j Ich mit on allin vechte Unde alle myn geslechte Keyner dich ubirwindin mag Wannen du bist mir eyn wedirslag Ou we mynes gesellin 10 Gut ist dure gefelle.

Hier und v. 7 bietet $G$ offenbar das richtige.

Es mögen noch einige abweichende lesarten von $G$ folgen. 24. Id is my nw gevallen. 26. nicht szer guth. 27, 28. - desse rede dy ick em gerne dede. 29. - van em to hande. 31.32. Alzo is my von dy geschen Hir vmme mót ick van dy tihen. 34. Ick volgede gerne dynen rat na. 44. - sprack hen wedder sam. 47. Ick was wisz dat segge ick $d y$. 51. vordragen. 52. dar dy nicht nummer sagen. Nach 54: Do hüff dy dufel wedder an Dessze rede he began He sprack vnsze koningk. - 56. was. 63. mer 64. stan by $d y$. 70. glas. 72. in dat mer nat. 73. Babylon. 74. gulden lon. 78. dat isz der werlde vntrost. 86. hingk. 87. nicht andirs. 88. Opper vnsze gode Loueszam. 89. He spragk du salt erkennen. 90. Got wil ick dy nennen. 91. alle dingk. 94. an gode gar vordorffen. 97. Tyen aue die cleydere bloth. 98. wil vnd roth. 99. szere von tornen. 104. alzo eyne kercze. 105. van pync werde erloszt. 106. vnd van der hellen róst. 109. fehlt. Nach 110. Juwe radt der is eyn wicht. 115. eyne kope grot. Nach 116: He lith sie dar yn szencken Nicht ergers konde he erdenken. 121. Llosze my here von der sunder bant. Nach 124: U) sette my dat heil I)at van my der sunder teil (? Die vorlage hatte vielleicht: Inwach von mir der sunden meil).

Keine von beiden hss. kann, nach diesen abweichungen zu schliessen, den anspruch machen, den originaltext zu liefern: bald gibt die Göttinger, bald die Erfurter das richtige. Es ist offenbar noch eine ältere gemeinsame grundlage vorauszusetzen. Es fragt sich, welchem dialect und weiterhin, welcher zeit dieselbe angehörte.

$G$ weist einige nd. formen im reime auf: nd. $t$ im reime blot (bloss): rolh (aber hier bietet $\mathrm{E}$ mit der lesart wisz als eyn 
slosz sicher das richtige) und in vorgat (vergass): hat (hatte), grot: gebot. $\mathrm{Nd}$. $d$ in moder: broder, rede: dede (täte) ${ }^{* *}$; fermer die reime licht (liegt): frist, tornen: vorbornen, lave (laudi): klage, alzo: to, her (huc): er (ihr), my (dat. sing.): sie: syn: votelin.

Dem gegenüber steht aber eine nicht unbedeutende anzahl rein hochdeutscher reime: vorgas (vergass) wird einmal sogar so geschrieben im reime: was; herzustellen ist es im eingang v. 14 ebenfalls im reime: was (wie st. wart mit den andern hss. zu lesen ist); ebenso ist $h a z$ v. 30 in demselben reime herzustellen; geschrieben wird noch einmal das: was (=daz: was). Der nd. form tho (zu) widersprechen die reime derselben: bistu, meinestu, nu (2 mal). Statt der formen $m y d y$ für dat. u. ace. wird mir durch den reim: dir (tier), mich und dich durch den auf louelich, ewichlich gesichert.

$\mathrm{Nd} . k$ wird widerlegt durch maken: lachen, waken: trachten; nd. $t$ durch hercze: kercze.

Nd. kann also das original nicht gewesen sein, aber auch nicht hd., da der reim tornen: vorbornen, wie die übereinstimmung von $G$ und $E$ beweist, schon dem urtexte angehört hat. Der dialect desselben war ohne zweifel md.; darauf weisen auch reime wie sere:gebere und: swere; bringen (d.i. brengen): lengen (in $\mathrm{G}$ und $\mathrm{E}$ ); ferner das md. wort vullemunt (: munt), woraus selbst der Leipziger druck ein sinnloses wolmuth machte. Md. können auch reime sein, in denen $d$ statt hd. $t$ auftritt, sowie die formen licht (liegt)**) und labe (lobe).***)

Auch apokopierung des infinitiv $n$ kommt im reime vor, so: syen (sehen, l. sề) :gê (conj.) senden : ende; vielleicht gehörten auch die in $\mathrm{G}$ nicht enthaltenen reime E. 28, 34, 64 dem originale an. (Dass Schum a. a. o. s. 100 auch vorslinde als inf. auffuhrt, ist ein versehn.) Die wenigen speciell niederdeutsehen reime dürfen wir gewis dem schreiber von $G$ zusprechen, der auch sonst ziemlich willkürlich verfuhr, wie zahlreiche entstellungen des sinnes beweisen. Das er nicht einmal den dialect seiner vorlage gentigend verstand, beweist z. b. die lesart zu

*) Auch hier ist die lesart von $\mathrm{E}$ mit dem reime lêre : bekêre anzunehmen.

**) cf. z. b. Marien rosengarten (bei Bartsch erlüsung anhg. XIX.) v. 31 pflicht (=pfliget): gericht.

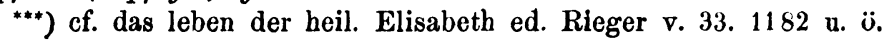


v. 47 genugsam (die vorlage hatte: waz ich weiz daz sage ich dir oder wie L., der schreiber fasste weiz als wîse und waz als was auf); auf ein ähnliches misverständnis scheint die textesentstellung bl $1^{\text {t }} 8$ zuruickzugehn. Das original hatte wol $i z$ en sî $d a z$. Merkwürdigerweise sind auch die beiden andern überlieferungen hier entstellt und v. d. Hagen gibt daher auch schon diese verbesserung an.

Was die zeit der abfassung betrifft, so verweisen sprache und reime mit gröster wahrscheinlichkeit auf das 14. jh. wie auch Schum vermutet - und wol noch auf die erste hälfte desselben. Unreine reime sind nicht gerade selten: draveden (trabeten): sageden, worden : vordorffen, hengen : henden; kraft: machl, vóp : bóck, visch : is (ist); wart : wort, an : hân : gedân, nôt : gol, wort : gehôrt. Selten ist noch apokope des $e$ nach langer stammsilbe (geist: leist praestabat) und unorganische dehnung im reim (noden : goden göttern, samen zusammen: amen, geneszen:kyszen). Diess sowie das gewicht, welches noch der tiefton im reim behauptet, spricht gegen spätere zeit. Beispiele sind tornich : wenich, sachmodich : dich, ja selbst forchten (dat. plur.): ynnichlichen*) wird gereimt. Auch reime wie sulverin : guldin, iszerin : kopperin dürften in späteren gedichten nicht vorkommen. Besonders häufig erscheint noch die endung md.'kerkenere, mere (mhd. muere): merterere : bichtigere; eine eigentümlichkeit, die sich besonders im Thüringischen dialect lange behauptete.

Wie unter den deutschen umdichtungen der Margaretenlegende die letzthesprochene, der zahl der hss. nach zu urteilen, dic verbreitetste war, so war unter den lateinischen versionen dic in des Mombritius samctuarium **) II, 104 ff. uberlieferte oftenbar die bekantituste und am meisten benutzte. Für Bartschs Margareten marter und für das Erfurter bruchstück hat Schum a. a. o. dieselbe bereits als quelle nachgewiesen. Die dem letzteren zu grunde liegende bearbeitung folgte derselben auch in

*) Dass aber deshalb nicht auch hier an ein original des 12. jhs. gedacht werden darf, beweist ausser den äusseren kennzeichen auch der stil des gedichtes, der in seiner unbehiilflichen breite, reich an plattheiten und herkïmmlichen flickwörtern für den reim, das gepräge der angegebenen zeit trïgt.

${ }^{* *}$ ) 2 bde. fol. Mediolani s. a. (vor 1480).

Beiträge zur gesclichte der deutschen sprache. I. 
den ubrigen nur in $G$ erhaltenen teilen in derselben weise. Meist schloss sich der dichter ziemlich sclavisch dem lateinischen texte an, aber natürlich bringt schon das reimbedürfnis einzelne abweichungen mit sich und man braucht deshalb noch nicht mit Schum die benutzung einer deutschen quelle neben der lateinischen anzunehmen. Die geringen ähulichkeiten dieser abweichungen mit stellen in der Margareten marter scheinen nur darauf zu deuten, dass der lateinische text, welcher den deutschen dichtern vorlag, nicht ganz identisch war mit dem so viel später bei Mombritius abgedruckten, was sich auch sonst nachweisen lässt. Einzelnes gewinnt übrigens auch ein anderes licht durch die mitgeteilten lesarten von $G$, vgl. die zu v. 7 und v. 10, welche zeigen, dass der dichter das lateinische nicht falsch verstanden habe.

Eigene zutat des dichters ist die einleitung. Der anfang der legende selbst wird getreu, zum teil wörtlich nach Mombritius berichtet, nur wird der bei Mombr. und in Hagens und Bartschs Margareta angegebene name des vaters der Margareta nicht erwälnt und ihr wird das alter von 12 statt von 15 jahren beigelegt. Margareta wird von ihrem vater, der sie hasst weil sie dem christentum zugetan ist*), einer amme ubergeben, deren schafe sie hitten muss. Dabei erblickt sie der christenfeindliche praefect (in G greve) Olybrius; er lässt von liebe zu ihr entflammt, die widerstrebende ergreifen. In dem nun folgenden gespräche zwischen ihm und Margareta, welches in $G$ (weniger in der Margareten marter) im einzelnen von Mombritius etwas abweicht, bekennt sich Margareta als christin und da alle uberredungsversuche des Olybrius scheitern, lässt dieser sie ins gefăngnis werfen. Nochmalige gutliche versuche des praefecten am nächsten morgen haben keinen bessern erfolg; die heilige wird aufgehängt und gepeitscht, ohne dass es gelingt ihron

*) An dieser stelle hat der schreiber einen recht gedankenlosen fehler gemacht, indem er bl. 2a v. 13 mitten im satze abbricht und uns mit einem male in das gespräch hinein versetzt, welches Margareta im kerker mit dem teufel hat; es geht dann im zusammenhang weiter bis 8 a 13 , wo die erzählung der jugendgeschichte fortgesetzt wird; dann unterbricht 20 b. 9 wider den zusammenhang, schliesst sich aber ganz genau an 8 a 12 an. Es ist also einfach der passus $8^{\text {a }} 13$ bis $20^{b} 8$ zwischen bl. 2 a v. 13 und v. 14 einzuschieben. 
glauben zu erschüttern. Eine darauf folgende noch grausamere zerfleischung, von der Mombritius, Margareten marter und der Leipziger druck berichten, wird in $\mathrm{G}$ - abgesehn von einer geringen andeutung - übergangen. Margareta wird abermals in den kerker geworfen und betet, dass ihr gott ihren feind zeigen möge. Die angabe bei Mombritius, dass Contimus - der gewährsmann der legende - das nun :folgende durchs fenster mit angesehn habe, wovon ohne nennung des namens auch die Marg. marter berichtet, fehlt wider in G. Es erscheint nun der teufel als drache $\left.{ }^{*}\right)$, der in folgender wunderlichen weise nach Mombr., abweichend von den übrigen deutschen bearbeitungen, in $G$ beschrieben wird.

G. fol. $14^{\text {b }}$ z. 3 ff.

Do sach sie hen vnd her.

Do sach sie in den kerckener

5 Den dufel sach sie vor sick sten $b$

Vth eynen vinstern winkel gen. $a$

He was gruwelich gar

Vnde mennigher varffe har:

Dy har weren suluerin,

10 De bart was em guldin.

Dy tene weren iszeren.

Syne ougen weren kopperin.

Vth syner neszen gingk eyn rock

Vnde eyn mechtich fuer ock, 15 Dar van eyn grot licht schen.
Mombr. $105^{a}$

Et ecce subito de angulo carceris exiuit draco horribilis: totus variis coloribus deauratus.

Capilli eius et barba aurea:

et videbantur dentes eius ferrei

Oculi eius uelut Margaritae splendebant

et de naribus eius ignis et fumus exibat [Lingua illius anhelabat. super collum eius erat serpens. gladius candens in manu eius videbatur et fætorem faciebat in carcere. traxit se in medium carceris : et sibilabat fortiter]. et factum est lumen in carcere ab igne: qui exibat de ore draconis.

Er verschlingt die heilige, welche das zeichen des kreuzes macht und ihn dadurch in zwei sticke spaltet. Es folgt ein dankgebet der Margareta, darauf die erscheinung des zweiten teufels in gestalt eines moren. Sie wirft ihn nieder und setzt ihm den fuss auf den nacken; eine taube erscheint vom himmel und bestärkt sie. Dann folgt die unterredung mit dem teufel,

*) Dass dies ungetüm ein drache gewesen, wird in G nicht ausdrücklich berichtet, möglicherweise absichtlich, da der bearbeiter sich einen drachen wol anders vorstellte. 
die ebenso wie das weiter sich anschliessende (uberwindung des teufels, nochmalige uberredungsversuche des Olybrius, feuerund wassermarter) auch im Erfurter bruchst. enthalten ist. Als Margareta, ins wasser geworfen, gebetet hat, dass ihr dasselbe zur taufe und zur sündenreinigung dienen möge, erfolgt ein erdbeben, welches ihre fesseln löst; eine taube bringt ihr vom himmel eine krone und, wie $G$ selbständig hinzusetzt,

dy werster hüffe ( $l$. westerh.)

Vnde dat ander westercleit.

Nach diesem wunder bekehren sich 5000 ohne weiber und kinder zum christentum. Olybrius befiehlt, die Margareta zu enthaupten; der henker, Malchus in den deutschen versionen, bei Mombr. Maleus*) genannt, gibt ihr frist zum beten und sie bittet, charakteristisch genug, für alle die ihre leidensgeschichte lesen und abschreiben, ihr lichte stiften, kirchen bauen u. s. w.; besonders soll in dem hause, wo man ihre passion hat, kein fehlerhaftes kind geboren werden. Es erscheint nun wider eine taube vom himmel und sagt ihr die gewährung zu. Der henker, der sich anfänglich geweigert und sie um verzeihung gebeten, enthauptet sie und fällt tot neben ihr nieder. Ein donnerschlag wirft alle umstehenden zu boden. Die engel kommen und führen ihre seele in den himmel, die teufel wehklagen, kranke werden an ihrem leichnam geheilt. Mit dem nach Mombr. widergegebenen gesang der engel schliesst $G$, abweichend von Hagens Margareta und dem wesentlich damit ubereinstimmenden Leipziger druck:

Dy engeln sungen eyn sangk, G fol. $22 \mathrm{~b}$ z. $6 \mathrm{ff}$.

Die in den wolken sere klangk.

Sie sungen alle hoch,

Dy sangk lude alzo:

10 „Du bist aller gute grot;

Die werlt steit an dynem gehot."

Vnd sungen denne noch mere

*) Malleus ist ein christlicher name des teufels ct. mythol. 559 wie das deutsche hämmerlein and hämmerling; die häufigere anwendung des deutschen wortes zur bezeichnung des henkers (cf. D. W.) legt die vermutung nahe, dass auch das lat. malleus dafür gebraucht sei; auch der wortlaut der stelle bei Mombr. II, 106 b macht es wahrscheinlich, dass das wort hier als appellativum zu fassen sei: Questionarii comprehenderunt eam: et duxerunt foris ciuitatem. Dixit Maleus ad eam: Extende ceruicem tuam. (Vorher ist das wort noch nicht vorgekommen.) 
Dorch dy godes ere:

„Here hiliger got,

$15 \mathrm{Du}$ bist geheiten sabaoth.

Vor vullet ist dat ertrick

Myt dynar zcirheit vnd dat hemelrick“.

Hir het dat bokelin eyn ende.

Got mote vns syne hulpe senden

5 Vnde geue vns allen samen

Dat ewyge hemmelricke. Amen.

Bei Mombritius folgt noch die bestattung der heiligen durch T'ectinus, der in der Margareten marter, die auch davon berichtet, Theodosius heisst. - In allen den angefuhrten hauptzügen der legende stimmen sonst diese beiden deutschen bearbeitungen mit dem Mombritius genau, teilweise wörtlich uberein, ohne unter sich in einem abhängigkeitsverhältnis zu stehn.

Soweit man aus den von Docen a. a. o. mitgeteilten proben aus der Margareta Hartwigs vom Hage einen schluss ziehen kann, entnahm auch dieser seinen stoff der bei Mombritius erhaltenen tradition. Man vergleiche den dort gegebenen eingang des gedichtes mit dem bei Mombritius:

Nach der zit daz warer got

Crist väterliches willen bot Gecrízet an der menschait starb

Ze helle do den rawp erwarb

u. s. w.
Post resurrectionem domini

nostri Jesu Christi et gloriosae

tempus ascensionis eius in

caelum ad patrem omnipotentem

etc.

ferner einzelne aus dem gedichte mitgeteilte sätze:

di mir die kirchen machen

oder ir licht mir brennen

qui basilicam in nomine meo fecerit et quisquis lumen fecerit in basilica mea de suo labore.

wer sie (die legende) schreibe lese et scripserit passionem meam uel qui oder ze schriben frume de suo labore comparauerit codicem passionis meae

endlich die s. 159 mitgeteilte stelle, wo das zauberbuch Mambre erwähnt wird mit Mombr:: In libris tamen ianuae et mambrae inuenies genus nostrum. scrutare et uide - - Nam uiae nostrac non sunt super terram sed cum uentis ambulamus. Die geschichte von der einsperrung der teufel durch Salomo wird fast wörtlich nach Mombr. erzählt. Der name des gewährsmannes aber wird, wie bei den andern deutschen bearbeitern, die denselben erwähnen, Theotimus, nicht wie bei Mombr. Tectinus oder Contimus und der teufel Belial, nicht Bezeleel, genannt.

Auch Wetzel benutzte für sein gedicht offenbar den text 
des Mombritius und nicht den der Acta SS. Freilich verfulır er mit seinem stoffe ganz anders als die übrigen bearbeiter. Er suchte denselben nicht einfach in reime zu bringen, sondern er wollte ihn wirklich poetisch umformen. Daher liess er es nicht an redegewanten und redseligen ausschmückungen fehlen, wie in der schilderung der zeit der christenverfolgung v. $73 \mathrm{ff}$. und in der im Hartmannschen stile ausgeführten rede des Olibrius v. $459 \mathrm{ff}$, wie in der ganz entsprechend gehaltenen antwort der Margareta v. $508 \mathrm{ff}$; aber im ganzen gange der erzählung folgt er doch treu seiner vorlage und wie er auch die einzelnen charakteristischen züge der lateinischen überlieferung verwertete, mag beispielsweise die vergleichung des gebetes der von den knechten des Olibrius bedrohten Margareta zeigen: v. 258 sie rief ze gote unde sprach beata Margarita inuocare coepit Christum ac dicere

„herre got, erbarme dich Miserere mei domine. miserere mei.

260 genaedeclîch über mich. verlius mîne sêle niet mit ungeloubiger diet

ne perdas cum impiis

animam meam [aut cum uiris sanguinum uitam meam]

herre got, getroeste mich fac me laetari semper in te domine daz ich mit freuden lobe dich, J. Chr. et te semper collaudare.

265 sô daz dîn reine güete vor flecken mich behüete. $\mathrm{Ne}$ permittas animam meam contaminari et ne polluatur fides mea.

herre got, behalt an mir

daz ich geloube von dir.

der bluome und der reine nam Non inquinetur corpus meum: Non miner megetlîchen scham, proiiciatur Margarita mea in lutum:

275 den ich dir einen wihte in mînes herzen bîhte, dô ich von êrst den sin gewan daz ich verkôs alle man, und den ich roine her hân brâht, non immutetur sensus meus a turpitudine unquam. et ab insipientia diaboli

280 lâ mir niht werfen in daz bâht keinen irdischen man, wan sîn mîn herze nieman gan. den engel du mir sende

verlîch mir dîne wîsheit daz mîn zunge sî bereit ze antwuirten dem rihtaere,

290 des tiufels wîzenaere.

mir ist gelich an dirre frist Video enim me ut ovem in medio 
daz schâf daz undern wolven ist. luporum ecce facta sum sicut passer der vogel sich mir gelîchet, in raetibus comprehensa.

den der vogelaere erslichet

auch waen sich mir gelîche et sicut piscis in bamo.

der visch swenne erkumtgevarn

dâ in bestricket daz garn.

u. s. W.

Es liessen sich noch viele weitere einzelheiten beibringen, doch ich glaube, die gegebene probe beweist gentigend, dass und wie Wetzel die bei Mombritius widergegebene quelle benutzte *).

Andere quellen haben Haupts und Schades Margareta, sowie die im Passional enthaltene. Der dichter des letzteren benutzte auch hier die legenda aurea, welche in manchen punkten wesentlich vom Mombritius abweicht. Es scheint als sei hier etwas kritik geübt: die erscheinungen der taube fehlen, die engel und teufel treten bei Margaretas tode nicht auf. Auch die scene mit dem henker wird ausgelassen. Die erscheinung des drachen wird nicht näher beschrieben; er verschlingt zwar die heilige und wird durch das kreuz getötet, aber der dichter fugt hinzu 330,52

sumeliche haben verhowen

an den meren disen strich und sprechen, ez si mislich swaz deme wurme ist geseit.

was auf die worte seiner quelle hinweist (bei Grässe s. 401) nIstud autem quod dicitur de draconis devoratione et ipsius crepatione, apocryphum et frivolum reputatur."

Doch scheint ihm auch hier wie in andern teilen des werkes noch eine andere version neben dem Jacobus a Voragine bekannt gewesen zu sein. Die zahl derer, welche sich nach Margaretens wunderbarer errettung vom ertrinken bekehren, wird im Passional nur auf 8 , in den andern deutschen und lateinischen versionen, - auch in der leg. aur. - auf 5000 angegeben.

Die quellen der legende bei Haupt und Schade weiss ich nicht nachzuweisen. Dass dem dichter der letztgenannten die

*) Auch an den meisten der von Bartsch mit dem texte der Acta SS. verglichenen stellen ist die übereinstimmung mit Mombritius grösser. 
"Margareten marter" vorlag, ist bereits erwähnt; seine hauptquelle blieb aber eine version, welche mit denen bei Mombritius, Petrus de Natalibus, Jacobus a Vor., Surius, Acta SS. nicht ubereinstimmt. Dagegen trifft sein gedicht in mehreren punkten mit dem Hauptschen "Margareten püechlîn“ zusammen. So darin, dass Margareta ins feuer geworfen, nicht mit fackeln verbrannt wird*) und der versuch, sie zu ertränken, in kochendem wasser gemacht wird. Im gegensatz zu Mombr. findet in beiden gedichten auch die erste marter schon statt ehe Marg. in den kerker geworfen wird, während allein im "püiechlîn" auch die beiden folgenden martern schon rordem berichtet werden. Bemerkenswert ist noch, dass das letztere auch den namen von Margaretens mutter - Eugênne - mitteilt, der sonst in keiner deutschen oder lat. version genannt wird. Der name des vaters ist, wic in den andern deutschen bearbeitungen, Theodosius, in tibereinstimmung mit Mombr., der leg. aur. und Petrus de Nat.; bei Surius, und in den Acta SS. heisst er Aedesius.

Soviel über die gereimten bearbeitungen der Margaretenlegende. - In der älteren zeit war dic poetische form für die behandlung legendarischer stoffe offembar die beliebtere, erst seit dem 15. jh. kam auch auf diesem gebiete die prosa mehr zur geltung und besonders die übertragungen der legenda aurea, die natirlich auch unsere legende enthalten, hatten sich einer grossen ausbreitung zu erfreuen, wie die zahlreichen alten drucke der "passionale" beweisen. Aus früherer zeit sind von der Margaretenlegende nur zwei prosabearbeitungen bekannt. Die eine ist in der Gräzer hs. des 14. jhs. aberliefert, welche auch die himmelfahrt Marien von Konrad v. Heimesfurt enthält. Herausgeg. von Diemer in den kleineren beiträgen (Wicner Sitzungsber. 1851. 2, s. 316). Der eingang ist gereimt (30 v. v.) und das verleitete Pfeiffer zu der unrichtigen angabe bei Hpt. 8, 157 "gereimte Margaretenlegende," was auch in Wackernagels lit. gesch. überging. Die eigentliche legende ist nur eine latinisierende prosaübersetzung des Mombritiusschen

*) Schade v. 255 ff. Hpt. v. 377 ff. Schum hat das letztere iiberschn indem er Germ. 1S, 106 angibt, die Hauptsche version kenne keine getrennte feuer- und wassermarter. 
textes; einige auslassungen und geringe abweichungen werden auch hier ler lateinischen vorlage zuzuschieben sein.

Die aweite prosabearbeitung ist die, welche Hermann v. Fritslar seinen heiligenleben einverleibte (bei Pfeiffer mystiker I, $155 \mathrm{ff}$ ). Sie schliesst sich im wesentlichen der legende aurea an. Eine abweichende angabe von der zeitfolge der martern, welche hier alle unmittelbar hintereinander zwischen der ersten und zweiten einkerkerung berichtet werden, und ein in der legenda nicht enthaltener zusatz am schlusse bestätigen jedoch die angabe Hermanns (s.4,15), dass er nach mehreren quellen arbeitete. Von dem „20 mîle von Rôme in Tuscanjen“ belegenen ,kastelle zu sente Margarêten “ berichtet er nach eigener anschauung.

Eine verkürzende bearbeitung der legenda aurea ist die von Holland (die legende der heil. Margareta altfranzösisch u. deutsch. Haniover 1863) mitgeteilte "Sand Margareta" eines passionals v. j. 1463. Auffällig ist hier nur bei der sonstigen genauen übereinstimmung mit der queHe, dass die wassermarter, abweichend von derselben, wie in Haupts und Schades Nargareta berichtet wird.

Ausserhalb Deutschlands scheint die Margaretenlegende als stoff poetischer wie prosaischer behandlung nicht weniger willkommen gewesen zu sein. Die älteste bearbeitung, welche sie in einer vulgärsprache erfahren hat, ist die ags. prosaische Passio Scae Margaretae virginis, welche der herausgeber, Cockayne (narratiunculae anglice conscriptae 1861), ca 1000 ansetzt. Noch dem ausgange des 12. jhs. gehört das alliterierende gedicht Seinte Marherete pe meiden ant martyr an, von Cockayne zusammen mit zwei mittelenglisehen gereimten Margaretenleben (.. Seinte Margarete pat holi maide" und "meidan Margarete") herauscregeben in der 13. publication der early english text-socicty. Von cinem spïteren gedichte gleiches inhalts (die abfassung wird 1463 angesetzt) werden nur die schlussverse - a. a. o. s. VIII. - mitgeteilt.

Die beiden ersten dieser bearbeitungen gehen nun unzweifelhaft auch auf die su viel henutzte lat. version des Mombritius zuriick. Die ags. prosalegende ist nur eine fast wörtliche iibersetzung derselben.

Man vergleiche zu dem bereits mitgeteilten eingang bei 
Mombritius den angelsächsischen: Efter \}äre provunge and päre æriste and päre vuldorfästan upastîgnesse ûres drihtnes hælendes cristes tô god (so) fäder ealmihtigum. - Nur selten kommen kleine auslassungen vor: die bemerkenswerteste ist die der erzählung des teufels von seiner einschliessung durch Salomo. Andere geringe abweichungen werden meist der mit dem drucke des Mombritius nicht ganz congruenten vorlage zuzuschieben sein, wie denn auch diese abweichungen zum teil mit denen der deutschen bearbeitungen tibereinstimmen*). - Auch die

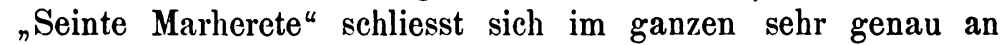
Mombritius an; nur machte sich auch hier der in alliterierenden gedichten so oft hervortretende und teilweise durch die form selbst begründete hang zu breiteren ausführungen geltend. Die gebete und reden - namentlich die rede des teufels, in welche der dichter auch eigene gedanken hineintrug**) - gehn noch bedeutend über die schon recht ausführliche quelle hinaus, so dass diess gedicht das umfangreichste unter allen bekannten Margaretenlegenden ist. Dass das gedicht ubrigens nicht etwa auf die ags. prosaübersetzung, sondern direct auf die lat. quelle zurückgeht, wird unter vielem andern auch durch die erzählung von Salomo und den teufeln bewiesen.

Schwerer ist zu entscheiden, auf welche grundlage die beiden gereimten legenden zuruckgehn. S. Margareta pat holi maide stimmt in der hauptsache mit der legenda aurea uberein. Ihr sind z. b. auch die worte des Olybrius entnommen, welche er an die von seinen knechten ergriffene Margareta richtet:

v. 75 pis tuo pinges pat $\mathrm{pu}$ nemnedest erst bicomep pe faire and suete

pat pu beo icome of heze blode and pat pu hote margarete

puse- tuo bicomep pe wel ynouz suche maide noble and freo

Ac pe pridde becomep pe nozt as pu mizt iseo****)

*) So hat z. b. gleich zu anfang die Gräzer prosalegende übereinstimmend mit der ags.: nach der marter und nach der urstende gegeniiber dem lateinischen post resurrectionem.

-*) besonders fol. 48a $7 \mathrm{ff}$.

***) Duo prima tibi recte conveniunt, quod nobilis haberis et pulcherrima margaritha comprobaris; sed tercium tibi non convenit. 
und der zweifel an der drachenerscheinung v. 165 Ac pis ne telle ic nozt forsope u.s. w.

Dagegen stinimt die scene vor Margaretas hinrichtung wider ganz mit Mombritius, der bericht der feuer-und der einen wassermarter aber mit der Hauptschen und Schadeschen Margareta überein, während sich die angabe, dass die leidensgeschichte sich 285 jahre nach Christi geburt unter Diocletian u. Maximian zugetragen habe, sonst nur in dem texte der Acta SS. *) findet; der bericht von 2 wassermartern ist dem dichter ganz eigentümlich. Derselbe wird wol schon als quelle eine zusammenstellung aus den verschiedenen versionen benutzt haben, schwerlich hat er sich seinen stoff aus denselben selbst zusammengesuchit. - Wider andere besondere zijge hat Meidan Margarete aufzuweisen, wie z. b., dass Margarete mit 7 andern kindern bei der amme aufgezogen wird u. a.; sonst ist hier Mombritius benutzt, aber in ziemlich freier weise und vielfach verkürzt.

Von französischen bearbeitungen sind handschriftlich mehrere in versen und in prosa vorhanden. Eine bruchstückweise in einer hs. aus dem anfang des 13. jhs. auf der bibl. zu Tours erhaltene Margaretenlegende ist nach du Méril (études sur quelques points d'archéologie et d'histoire littéraire Paris 1862, s. 228 u. 29.) von einem "sehr gewanten dichter des 12. jhs." verfasst. In den schlussversen, die du Méril (a. a. o.) mitteilt, nennt sich der verfasser Grace, worin dann du Méril ebenso wie Luzarche - Adam drame anglonormand s. XXXVIII. den Guace sieht $* * *$ ). Da der verfasser sich ebendort auf den lateinischen text des "Theodimus" beruft, so mag er auch nach Mombritius gearbeitet haben (_ - ce dit Grace qui de latin en romans mist ce que Théodimus escrit).

Vollständig veröffentlicht ist meines wissens nur das gleichfalls gereimte Margaretenleben einer Neuenburger hs. von Holland (die legende der heil. Margarete altfranzösisch u. deutsch). Diess hat viele, hie und da selbst wörtliche übereinstimmungen mit Mombritius. So im anfang:

*) hier jedoch mit abweichender zeitbestimmung. I. s. 12 .

**) vgl. du Méril la vie et les ouvrages de Wace in Eberts jahrb. 
Apres la sainte passion

Jhesucrist, a l'escension

Quant en son ciel fust monte etc.

vgl. den eingang bei Mombr. Daneben aber übergeht das gedicht nicht allein bedeutende in dieser version enthaltene stiucke - so die ganze feuer - und wassermarter - sondern es hat auch einzelne abweichungen aufzuweisen, die sich nicht wol erklären lassen ohne die annahme, dass nebenher noch eine andre bearbeitung oder, was wahrscheinlicher ist, dass eine verkürzte umarbeitung des Mombritiusschen textes benutzt sei.

Nach Bartsch, germ. stud. I, 8 anm., wird ubbrigens ${ }_{n}$ dieser altfranzösische text noch heute als volksbuch gedruckt und verkauft".

Die hss. der übrigen französischen bearbeitungen der legende sind aufgeführt bei du Méril und Holland a.a. o. VI. VII.

Auch eine niederländische Margaretenlegende in versen ist uns erhalten in dem bruchstücke der hs. eines passionals, welches vom ungenannten herausgeber des stuckes im Bclgisch museum voor de nederduitsche taal-en letterkunde I, $227 \mathrm{ff}$. ungefähr in die mitte des 13 . jhs. gesetzt wird. Das gedicht, nur $\left.178 \mathrm{vv} .^{*}\right)$, ist eine verktirzende umarbeitung des in der legenda aurea widergegebenen textes; neben einigen auslassungen beschränken sich die abweichungen im wesentlichen darauf, dass statt der feuermarter eine 3. zerfleischung, anders als in allen tibrigen bearbeitungen, berichtet wird. Dafur kommen aber auch oft genug wörtliche übereinstimmungen mit der quelle vor. Unmittelbar auf die Margaretenlegende folgt in der hs. die legende von der heil. Regina, welche hier sowol wie in der lat. quelle mit der ersteren eine so grosse ähnlichkeit zeigt, dass sie nur eine nachbildung derselben zu sein scheint.

Ich fasse noch einmal das resultat obiger untersuchung zusammen, soweit sich diese auf das verhältnis der verschiedenen bearbeitungen der legende bezog. Die in Hagens grundr. 229 und in den altd. wäld. III, 156 angeführten hss., das Erfurter bruchstiick, der Leipziger druck und die Göttinger hs., aller wahrscheinlichkeit nach auch die im anzeiger v. j. 1861, s. 391 und

*) anfang:

Passie ende sware verdriete

Doghede die rene Margariete

Ende al omme ons heren minne 
bei Hoffman Wiener hss. 90, 2 bemerkten hss., sind insgesammt auf eine md. gereimte Margaretenlegende des 14. jhs. zurtickzuführen. Diese sowic die in der Prager und der Klosterneuburger hs. erhaltene Margareten marter, die dichtungen Wetzels und Hartwigs, die Gräzer prosalegende und die beiden ags. bearbeitungen gehn direkt auf die bei Mombritius mit geringen abweichungen widergegebene lateinische legende zurtick. Ebenfalls benutzten dieselbe, aber mit beimischung aus verschiedenen fremden quellen, Hollands altfranzösische Margareta und die altenglische meidan Margarete. Eine selbständige stellung nimmt Haupts Margareten püechlîn ein; etwa zwischen ihm und der Mombritiusschen gruppe steht die niederrh. Margareten passie.

Das Passional, Herman von Fritslar, die bei Holland mitgeteilte deutsche prosalegende, Margareta pat holi maide und die niederländische Margareta schöpfen aus der legenda aurea; alle jedoch mit entlehnung einzelner zugge aus andern versionen.

Ich glaubte dem schon so reichlich angehäuften stoffe taber die Margaretenlegende nicht noch neuen hinzufügen zu dürfen ohne einen versuch, das vorhandene einigermassen zu sichten. So unbedeutend auch an sich die bearbeitungen der legende teilweise sein mögen: für die literaturgeschichte sind sie doch gerade in ihrer grossen anzahl von wert als ein sprechendes zeugnis für die geistes- und geschmacksrichtung ihrer zeit. Jenes wolgefallen des mittelalters am wunderbaren, ja unnatürlichen vereinigte sich mit dem glauben an den sehr realen nutzen des heiligencultus, um demselben an sich so unbedeutenden stoffe fünf jahrhunderte hindureh für das ganze abendland immer neue anziehungskraft zu verleihen. Und in so verschiedener gestalt die lateinische legende uberliefert war: uberall ist es doch gerade die wunderreicheste und unglaublichste version - die bei Mombritius -, auf welche vollkommen unabhängig von einander der geschmack der dichter verfältt. Erst später macht ihr die nüchternere legenda aurea den rang streitig, als man bei der behandlung des grossen legendencyclus der kürzeren fassung den vorzug gab.

Aber auch der inhalt der legende an sich ist in gewisser beziehung nicht ohne interesse: er zeigt, wie sich schon in fru- 
her zeit*) auch im christlichsten gewande orientalische mythen ins abendland einschlichen, wo sie dann gewis nicht wenig zur ausbreitung des verhängnisvollen zauberei- und hexenglaubens beitrugen. Mohammedanischen ursprunges ist die geschichte von der etnschliessung der teufel durch Salomo. Ganz ähnlich wie in unserer legende wird im Suleimanname nach v. Hammer rosenöl I, 221 dieser mythus erzählt: Salomo, dem die gewalt über alle dämonen gegeben ist, zwang die einen zum tempelbau indem er ihnen sein siegel auf den hals druickte, die andern aber, die sich ihm nicht unterwerfen wollten, bezwang er mit gewalt und sperrte sie in weinschläuche, flaschen und eherne töpfe, die er mit eigner hand versiegelte und auf den grund des meeres warf. Wie dann einer wider frei geworden ist darüber sollen "die wahrhaften geschichten der 1001 nacht" aufschluss geben. Vielleicht ist damit die erzählung vom fischer gemeint, der einen solchen von Salomo in ein gefäss eingeschlossenen geist befreit. Hier sind die Babylonier die unvorsichtigen befreier der teufel ${ }^{* *}$ ): Babylonien ist das heimatland der ${ }_{n}$ schwarzen kunst" und die teufel selbst sprechen chaldäisch. (vgl. Roth über den zauberer Virgilius Germ. IV, 278). Wie diese geschichte von den eingesperrten teufeln in die Virgiliussage eingang fand (Roth a. a. 0. 277 u. 78), so vermutete Docen noch einen anderen, directeren bezug unsrer legende auf jene sage. In den büchern Jamne und Mambre, auf welche nach der version des Mombritius der teufel die Margareta verweist, um näheren aufschluss uber die höllenbewohner zu erhalten, sieht Docen (altd. wäld. III, 159) „eine beziehung auf den zauberer Virgilius und auf jene so dunkelen verse im Wartburgkriege, die so trefflich durch den herzog

*) Die ags. prosalegende ca 1000; hss. der lat. legonde - wahrscboinlich in der Mombritiusschen version - aus dem 11.jh. vgl. Diem. a.a.o, s. 315 , text society $13 \mathrm{~s}$. VII.

**) Etwas abweichend wird dieselbe geschichte im Reinfrid ed. Bartsch v. 21042 ff. erzählt:

künc Salamôn hât ouch für wâr mit sîner künste meisterschaft verwürket aller tinvel kraft die fuoren in den lüften. ir tiuvellîchez güften was ouch verwïrket in ein glas. daz glas hôh gehenket was ûf in des tempels krône unz die von Babilône sich an den juden râchen. daz glas sî dô zerbrâchen und wânden dinne vinden golt. 
Reinfrit aufgeklärt werden". Er kann damit nur die erzählung vom zauberbuch des Zabulon oder Savilon meinen (Wartburgkrieg Simrock 156 ff. Reinfrid v. 21034 ff'), welches Christi geburt hintertreiben sollte, dann aber vom magnetberge, wo es auf wunderbare weise bewacht war, durch den Virgilius entführt wurde. Aber das steht mit dem in der Margaretenlegende erwähnten in gar keinem zusammenhang. Jannes und Mambres sind nach 2. Timoth. 3, 8 die zauberer, welche des Moses zeichen vor Pharao nachmachten. Eine alte lateinische aufzeichnung die auch ins ags. ubbersetzt wurde - mitgeteilt bei Cockayne narratiunculae s. 50 u. 67 - berichtet weiter, dass Mambre dafür in die hölle gekommen ist. Mit hülfe seines zauberbuches wird er dann durch seinen bruder Jannes auf die oberwelt heraufbeschworen, um diesem von den schrecken der hölle zu berichten und ihn vor der strafe nach dem tode zu warnen*). Das gemeinsame dieser erzählung mit dem berichte der legende ist also die tatsache, dass man glaubte, durch das buch Mambre**) aufschluss über die geheimnisse der hölle erhalten zu können.

Auf fremdem einflusse beruht auch ohne zweifel die beschreibung des als drache erscheinenden teufels. Diess ungetüm mit silbernem haar, goldenem bart, eisernen zähnen und perlen-oder kupferaugen hat nicht die geringste ähnlichkeit mit dem äusseren, welches sonst das mittelalter dem teufel oder einem drachen beilegt. Weun man die heimat der legende berticksichtigt, so wird man sich kaum der vermutung erwehren können, dass diese züge einem orientalischen götzenbilde entnommen seien***), wie denn ja bekanntlich die kirche so manchen heidnischen gott als teufel fortleben liess.

*) Zu den sammlungen Liebrechts in Gervasius s. 87 und Germ. IV, 371 „das grab und seine liinge" liefern einen beitray die nur in der ags. uibersetzung vollstaindig erhaltenen schlussworte des Mambres: and äfter pam pe pu deád bist ponne cymst pu tô helle and betvix deádum mannum bið pîn eardingstôv nið̉er on eorðan and pîn seảd bið tregea cubita vîd and feóvra lang.

**) vgl. auch Fabricius cod. pseudepigr. vet. test. I, 813 ff. u. bes. s. 818 .

***) Ueber den einfluss bildlicher darstellungen heidnischer gottheiten auf die mittelalterliche vorstellung vom aussehn des teufels vgl. Mentzel christl. symbolik unter teufel.

GÖ'TIINGEN.

FRIEDRICH VOG'T. 